\title{
Competitive balance in the big five European leagues based on Shannon entropy
}

\section{Yang-Qing Zhao', Hui Zhang ${ }^{1}$}

1. Department of Physical Education,Zhejiang University,Hangzhou,China

This preprint has been removed because it was erroneously supplied by a journal. 